Erschienen in: Konopka, Marek/Strecker, Bruno (Hrsg.): Deutsche Grammatik

- Regeln, Normen, Sprachgebrauch. - Berlin, New York: de Gruyter, 2009. S.

141-165. (Institut für Deutsche Sprache. Jahrbuch 2008),

https://doi.org/10.1515/9783110217360.3.141

\author{
Wolf Peter Klein
}

\title{
Auf der Kippe? \\ Zweifelsfälle als Herausforderung(en) für Sprachwissenschaft und Sprachnormierung
}

\begin{abstract}
Auf der Basis einer spezifischen Definition des Begriffs „sprachlicher Zweifelsfall“ und einiger einführenden Bemerkungen zu potentiellen Entstehungsursachen wird im Text zunächst erläutert, in welchen Hinsichten sich die Sprachwissenschaft bisher mit diesen sprachlichen Einheiten beschäftigt hat. Dabei wird die These vertreten, dass die Zweifelsfälle aus verschiedenen Gründen traditionell eher marginalisiert wurden. Nachdem mit konditionierten und unkonditionierten Zweifelsfällen zwei Haupttypen unterschieden worden sind, widmet sich der Text der Sprachnormierung von Zweifelsfällen. Der Ausgangspunkt liegt darin, die geforderte Sprachberatung als argumentativ orientierten, rationalen Umgang mit sprachlichen Zweifelsfällen zu begreifen. Dazu wird eine systematische Entscheidungsprozedur entworfen, die als Grundlage für die Klärung von Zweifelsfällen fungiert. Mit dieser kleinen Theorie der Sprachnormierung von Zweifelsfällen wird auch das Ziel verfolgt, auf deskriptiver Basis empirisch legitimierte Sprachnormen zu formulieren und damit nicht zuletzt einen sprachwissenschaftlichen Beitrag zur Formung des öffentlichen Sprachbewusstseins zu leisten.
\end{abstract}

Sprachliche Zweifelsfälle stellen seit jeher einen Gegenstand dar, bei dem normative Probleme des Sprachgebrauchs in besonders dringlicher Form zum Tragen kommen. Von daher ist es wohl unmittelbar verständlich, dass bei einer Tagung mit dem Untertitel „Regeln, Normen, Sprachgebrauch“ über dieses Thema ein Vortrag gehalten wird. Was Regeln sind, wie (kodifizierte) Normen angesichts des herrschenden Sprachgebrauchs sinnvollerweise beschaffen sein müssten und welche Rolle in diesen Zusammenhängen der Sprachgebrauch spielt, lässt sich am Beispiel der Zweifelsfälle sehr perspektivreich entwickeln.

Vor diesem Hintergrund soll in diesem Beitrag zunächst skizziert werden, was man unter sprachlichen Zweifelsfällen verstehen kann und in welcher Art und Weise sie bisher von der Sprachwissenschaft konzeptualisiert und erforscht wurden. Aufbauend auf dieser Vorklärung wird im zweiten Teil die Normproblematik in den Vordergrund gerückt. Damit ist auch die Feststellung verbunden, dass und wie sprachliche Zweifelsfälle im Rahmen eines rationalen Entscheidungsprozesses geklärt werden könnten. Das Ganze zielt nicht zuletzt darauf, sprachliche Zweifelsfälle als etwas zu begreifen und zu 
bearbeiten, das im Schnittpunkt von Sprachwissenschaft und öffentlichem Sprachbewusstsein angesiedelt ist. An diesem Punkt müssen - und das ist in dieser Kombination vielleicht etwas ungewöhnlich - sowohl Grundlagenfragen der Sprachwissenschaft diskutiert als auch praktische Sprachprobleme von linguistischen Laien gelöst werden.

Der Begriff „sprachlicher Zweifelsfall“ taucht in den gängigen sprachwissenschaftlichen Fachwörterbüchern nicht auf. Um also Missverständnisse zu vermeiden, möchte ich ihn wie folgt definieren:

Ein sprachlicher Zweifelsfall liegt dann vor, wenn (kompetente) Sprecher kommunizieren, im Blick auf die eigene Sprachproduktion (plötzlich) ïber verschiedene sprachliche Möglichkeiten (Varianten) nachdenken und sich nicht (einfach) für eine der bewusst werdenden Möglichkeiten entscheiden können.

Es ist an dieser Stelle nicht möglich, auf alle (teilweise durchaus problematischen) Gehalte dieser Definition einzugehen. ${ }^{1}$ Es seien lediglich einige Erläuterungen festgehalten. Zweifelsfälle bestehen also immer aus mindestens zwei Sprachformen (Varianten), die anhand bestimmter Sprachgebrauchssituationen identifiziert werden. Es ist hervorzuheben, dass ausdrücklich von verschiedenen sprachlichen Möglichkeiten die Rede ist. Mit anderen Worten, es geht nicht um die Reflexion über inhaltliche Aussage-Alternativen und das diesbezügliche Zögern. Wer nicht weiß, ob er das Bild eines Künstlers als gelungen oder misstungen, als schön oder sehr schön bezeichnen soll, hat sich inhaltlich noch nicht entschieden; ein sprachlicher Zweifelsfall liegt hier nicht vor. Bei sprachlichen Zweifelsfällen muss der semantische Gehalt demnach in einer vorläufigen sprachlichen (!) Form bereits identifiziert sein. Konstitutiv für sprachliche Zweifelsfälle ist also die Existenz von (mindestens) zwei sprachlichen Varianten („Möglichkeiten“) ( $a$ oder $b$ oder $c \ldots$ ), über die ein Sprecher bewusst ins Zweifeln gerät und die insofern als Stolpersteine der Kommunikation erscheinen. ${ }^{2}$

Der Ausgangspunkt bei der Erörterung sprachlicher Zweifelsfälle liegt, wie skizziert, bei konkreten Sprechern, die sich in konkreten Kommunikationssituationen (= Zweifelsfallsituationen) befinden und die konkrete Inhalte versprachlichen wollen. Es handelt sich um eine Perspektive, die - sprachtheoretisch genommen - von der parole, nicht der langue ausgeht und zunächst also eine konstitutiv pragmatische Blickrichtung besitzt. Die Eigenschaften tat-

\footnotetext{
Vgl. zu einer etwas ausführlicheren Klärung Klein 2003/2004.

2 Selbstverständlich gibt es gelegentlich Übergänge, bei denen die Frage nach dem Vorliegen sprachlicher oder inhaltlicher Alternativen nicht einfach zu entscheiden ist. Ferner vernachlässige ich an diesem Punkt die Frage, ob es auch als Zweifelsfall gelten sollte, wenn man lediglich über eine sprachliche Variante nachdenkt, ohne bewusst eine konkrete Alternative im Sinn zu haben. Wie der Ausdruck Zweifelsfall schon zeigt, gehört es jedenfalls zur Prototypik des Zweifels, dass zwei mehr oder weniger konkret umrissene Varianten in den Aufmerksamkeitsmittelpunkt geraten.
} 
sächlicher Zweifelsfallsituationen fungieren als Bestimmungsmomente der Definition. Sprache wird somit konstitutiv aus Laienperspektive konzeptualisiert. In diesem Zusammenhang sei hier auch festgehalten, dass selbstverständlich nicht aus jeder entsprechenden Zweifelsfallsituation die Existenz eines sprachlichen Zweifelsfalls abgeleitet werden kann. Denn um von einem sprachlichen Zweifelsfall im Deutschen zu sprechen, muss empirisch sichergestellt sein, dass es nicht um eine isolierte, einmalige Zweifelsfallsituation geht. Nur wenn eine nennenswerte Anzahl von Sprechern im Deutschen immer wieder an entsprechenden Punkten in Zweifel gestürzt wird, deutet ein solcher Befund auf das Vorliegen eines sprachlichen Zweifelsfalls hin. Wer sprachliche Zweifelsfälle zweifelsfrei und wissenschaftlich identifizieren möchte, muss demzufolge umfangreiche empirische Erhebungen anstellen.

Was die Existenz sprachlicher Zweifelsfälle angeht, sind im Überblick einige generelle Entstehungsfaktoren festzumachen. Sprachliche Zweifelsfälle resultieren in weiten Teilen aus Schriftsprachlichkeit, vor allem dann, wenn es sich bei der jeweiligen Schriftsprache um eine sog. „Kultursprache“ mit großer Tradition handelt. Situationen, in denen Sprecher im oben erwähnten Sinn in Zweifel gestürzt werden, ergeben sich insbesondere bei der Produktion schriftlicher Sprache. Zum einen führen Schreibprozesse ohnehin häufig und sozusagen naturwüchsig zu verschiedenartigen Formen bewusster Sprachreflexion, zum anderen ist schriftliche Sprache in bestimmter Hinsicht digital organisiert. Letztere Eigenschaft fördert die Entstehung sprachlicher Zweifelsfälle, insofern mehr als zwei sprachliche Zwischenstufen und graduelle Übergänge oft nicht als solche abgebildet werden können, sondern im Blick auf lediglich zwei Möglichkeiten entschieden werden müssen. ${ }^{3} \mathrm{Um}$ einige einfache Beispiele anzusprechen: Zwischen Groß- und Kleinschreibung gibt es keine dritte Möglichkeit, auch wenn die grammatischen Randbedingungen darauf hinweisen, dass bestimmte Fälle als Übergangserscheinungen mit mehreren Zwischenstufen zu charakterisieren sind. Wer an bestimmten Punkten zwischen der nominalen Flexionsendung $-m$ und $-n$ oder zwischen singularischer und pluralischer Subjekt-Prädikat-Kongruenz schwankt, wird dieses Schwanken, das als solches (!) im Sprachsystem selbst verankert sein kann, in der Orthographie oft nicht abbilden können. Er muss sich vielmehr zwischen den beiden Varianten entscheiden, die das Schriftsystem bzw. die Grammatik jeweils zur Verfügung stellt. In derselben Weise ist es nicht möglich, zwischen einer umgelauteten und einer nicht-umgelauteten Komparativform eine dritte Möglichkeit zu wählen, bei der diesbezügliche Zweifel getilgt sein würden.

Eine weitere Existenzursache, aus der sprachliche Zweifelsfälle resultieren, liegt in der Varietätenvielfalt einer Sprache. Ganz generell lässt sich wahrscheinlich eine einfache Korrelation formulieren: Je mehr (soziale, funktionale, regionale, historische, stilistische) Varietäten es in einer Sprache gibt,

3 Dazu und zum folgenden vgl. v. a. Bredel 2006 sowie die Beiträge im Heft, zu dem Bredel 2006 die Einleitung formuliert. 
desto mehr sprachliche Zweifelsfälle werden entstehen. Derartige Fälle besitzen allerdings einen anderen Charakter als die Zweifelsfälle, die oben im Zusammenhang der Schriftsprachlichkeit in den Blick genommen wurden. Denn das mit der Vielheit der Varietäten verbundene, enorm umfangreiche Sprachwissen ist nicht einfach homogen über die Sprachgemeinschaft verteilt. Diese ungleiche Verteilung kann dann gelegentlich dazu führen, dass Sprecher bestimmte Varianten zwar (dunkel und bruchstückhaft) kennen, die jeweiligen Gebrauchsbedingungen aber nicht so weit überblicken, dass sie sich ohne Zweifel für eine der bewusst werdenden Varianten entscheiden könnten. Zugespitzt formuliert: Vielheit und Mannigfaltigkeit kann Desorientierung und Zögern bewirken.

Dazu kommt, dass in komplexen sprachkulturellen Kontexten oft spezifische Formen metasprachlichen und sprachdiskursiven Bewusstseins geschaffen und tradiert werden, etwa in Normschriften oder in sprachdidaktischen und sprachkritischen Diskussionszusammenhängen. Diese metasprachlichen Wissensreservoire bewirken nicht selten, dass Sprecher über ihre Sprache reflektieren und dabei in einigen Fällen auch in Zweifel über bestimmte Wörter, Wortformen oder syntaktische Konstruktionen gestürzt werden. Zuletzt sei darauf hingewiesen, dass Formen höherer sprachlicher Komplexität zu (sprachsystematisch bedingten) Regelkonflikten führen können, die sprachliche Zweifelsfälle hervorrufen. Dazu gehören beispielsweise auf syntaktischem Feld bestimmte Formen (mehrfacher) Sub- oder Koordination, die notwendigerweise unklare Rektions- oder Kongruenzverhältnisse schaffen und damit die Sprecher bei der Auswahl entsprechender sprachlicher Formen irritieren. ${ }^{4}$

Nimmt man nun sprachliche Zweifelsfälle, wie oben definiert, in den Blick, so lässt sich die Tatsache konstatieren, dass sie in der Gegenstandskonstitution moderner Sprachwissenschaft bisher eher eine untergeordnete Rolle spielten. Sie wurden, wenn überhaupt thematisiert, lediglich als randständige Objekte angesehen und insofern als solche kaum näher untersucht. Diese Vernachlässigung hat Gründe, die ich kurz ansprechen möchte, da sie den grundsätzlichen sprachwissenschaftlichen Zugang zu diesem Phänomenbereich betreffen. Insgesamt arbeitet die Sprachwissenschaft seit dem 19. Jahrhundert eher theorie- als praxisorientiert. Damit geht ein Desinteresse an Normfragen und Themen mit didaktischem, sprachkonsultativem Horizont einher. Symptomatisch dafür sind bereits die emphatischen wissenschaftstheoretischen Bemerkungen Jacob Grimms im Vorwort seiner Deutschen Grammatik. Nachdem er in Absetzung von einer langen, mächtigen (Grammatik-) Tradition ausgeführt hatte, dass die Sprache nicht „unter die gegenstände des schulunterrichts" gerechnet werden sollte, spitzte er seine Position folgendermaßen

4 Vgl. z. B. Klein 2004b sowie - allerdings mit ganz anderer konzeptioneller Stoßrichtung - auch Fanselow/Féry 2002. 
zu: ,jeder Deutsche, der sein deutsch schlecht und recht weiß, d.h. ungelehrt, darf sich [...] eine selbsteigene, lebendige grammatik nennen und kühnlich alle sprachmeisterregeln fahren lassen." (Grimm 1818, S. 139)

Muttersprachliche Sprecher sind für Grimm also, um es modern auszudrücken, in allen Situationen unmittelbar kompetent; sie bedürfen daher auch keiner Orientierung (von außen). Unter dieser Prämisse fallen Zweifelsfallsituationen aus dem Untersuchungsbereich der Sprachwissenschaft heraus. Sie werden geradezu wegtheoretisiert. Wohlgemerkt, ich möchte damit nicht behaupten, dass es für diese romantische, für die Geschichte der (germanistischen) Sprachwissenschaft äußerst folgenreiche Auffassung Grimms nicht gute Gründe gäbe. Natürlich muss man in solchen Bemerkungen zurecht den Beginn der modernen, nicht-normativen Sprachwissenschaft sehen. Allerdings ergibt sich diese Wissenschaftlichkeit eben auch durch eine Abstraktion vom tatsächlichen Sprachverhalten, durch die Zweifelsfälle von vorneherein vernachlässigt werden. In der Folge derartiger Sprachtheoreme wird im übrigen auch eine nachdrückliche Dissoziation zwischen Sprachwissenschaft und öffentlichem Sprachbewusstsein (inklusive sprachdidaktischer Fragestellungen) eingeleitet (Dieckmann 1991). Durch diese Entwicklung, die im Gegensatz zur recht praktischen sprachwissenschaftlichen Orientierung der vorhergehenden Zeit steht, ist bekanntlich auch die Gegenwart immer noch geprägt.

Die Tendenz, Zweifelsfälle als Gegenstand der Sprachwissenschaft eher auszublenden als zu fokussieren, wird überdies durch die grundsätzlichen Randbedingungen moderner grammatischer Arbeit verstärkt. Der empirische Ausgangspunkt für die Konstruktion von Grammatiken sind - wie könnte es anders sein? - eindeutige, unzweifelhafte Grammatikalitätsurteile. ${ }^{5}$ Aufbauend auf klaren Basisstrukturen müssen zunächst fundamentale grammatische Verhältnisse aufgearbeitet werden. Erst im Verlaufe der weiteren Untersuchung können gegebenenfalls „Schwankungen“ und „Varianten“ thematisiert werden. Mit anderen Worten, es liegt sozusagen in der Natur der grammatischen Reflexion, dass zweifelhafte Fälle eher ausgeblendet werden. Ganz Ähnliches gilt für die einflussreiche Annahme eines „idealen SprecherHörers", der durch eine mehr oder weniger homogene und allumfassende Kompetenz ausgezeichnet sein soll. Diese theoretische Konstruktion dominierte bekanntlich viele jüngere Arbeiten der Sprachwissenschaft und muss, zumindest in Teilen, als eine durchaus gerechtfertigte, fruchtbare Abstraktion gelten. ${ }^{6}$ Dass reale Sprecher aber längst nicht in jeder Situation „kompetent““

Dieser wissenschaftstheoretische Zugang wurde beispielsweise in der amerikanischen Tradition bei N. Chomsky als „Prinzip der klaren Fälle“ terminologisiert (Reis 1979), besitzt faktisch aber auch in anderen sprachwissenschaftlichen Schulen eine anhaltend große Bedeutung.

6 Neuere Arbeiten wollen in diesen Traditionen allerdings erfreulicherweise den Begriff der Graduiertheit grammatiktheoretisch fruchtbar machen und richten sich im Zeichen der sog. Optimalitätstheorie auch gegen den hergebrachten binären Charakter von Grammatikalitätsurteilen (z. B. Schütze 1996, Fanselow u.a. 2006, Aarts 2007). Meines 
kommunizieren und „ideal“ funktionieren, also z.B. durch Zweifelsfälle tangiert werden, kann angesichts solcher theoretischer Annahmen kaum angemessen in den Blick geraten.

Die, wie oben angedeutet, ohnehin bestehende Kluft zwischen öffentlichem Sprachbewusstsein und Sprachwissenschaft wird durch diese Vorgaben noch einmal vertieft. Denn das öffentliche Sprachbewusstsein geht gerade nicht von den eindeutigen, klar klassifizierbaren sprachlichen Basisstrukturen aus. Sie sind für Nicht-Linguisten kaum von Interesse, weil sie normalerweise weder mit kommunikativen Problemen verbunden sind noch „interessante“ Sprachthematisierungen erlauben. Stattdessen spielen in den nicht-linguistischen Sprachdiskussionen von Anfang an gerade sprachliche Varianten und Schwankungen eine besonders bedeutende Rolle, also genau das, was die Grammatik aus guten Gründen zunächst ausblendet und nur in speziellen Fällen weiterverfolgt. ${ }^{7}$ Mit anderen Worten, das prekäre Verhältnis zwischen öffentlichem Sprachbewusstsein und Sprachwissenschaft wurzelt nicht zuletzt darin, dass man auf der einen Seite genau das aufgreift, was man auf der anderen Seite gerade in den Hintergrund stellt. Kein Wunder also, dass es auf diesem Feld immer wieder zu Missverständnissen, Ungereimtheiten und Inkompatibilitäten kommt.

Zuletzt sei darauf hingewiesen, dass sprachliche Zweifelsfälle auch deshalb bisher kaum adäquat sprachwissenschaftlich aufgearbeitet wurden, weil sie im Übergangsbereich zwischen System- und Soziolinguistik angesiedelt sind. So fehlt den systemlinguistischen Perspektiven auf entsprechende Phänomene oft ein Gespür für die sozial(psychologisch)e Relevanz der Thematik, während in soziolinguistischen Perspektiven die systemlinguistischen Hintergründe mancher Zweifelsfälle nicht selten unter den Tisch fallen. Wer Zweifelsfälle aber nur aus systemlinguistischer oder nur aus soziolinguistischer Sicht in den Blick nimmt, verfehlt eine angemessene linguistische Thematisierung, die möglichst viele Elemente der Sprachwirklichkeit aufgreifen würde.

Damit wiederum mag es auch verbunden sein, dass Begriffe wie „Sprachschwankung" und "Sprachvariation" trotz ähnlichen referenziellen Angriffspunkten gegenüber dem Begriff „Zweifelsfall“ deutlich bevorzugt wurden. Diese Präferenz kann man als symptomatisch für die Zurückhaltung interpretieren, sprachliche Zweifelsfälle per se zum sprachwissenschaftlichen Gegenstand zu erheben. Stattdessen soll mit distanzierenden Begriffen wie

Erachtens bleibt freilich abzuwarten, ob damit tatsächlich eine grundlegend neue, konstitutiv empirische Orientierung der Sprachwissenschaft auf den Weg gebracht wird, in denen Zweifelsfälle in allen relevanten Dimensionen eben als Zweifelsfälle analysiert und aufgearbeitet werden.

7 Um ein Beispiel zu nennen: Kaum ein linguistischer Laie wäre wohl darüber irritiert oder fände es interessant zu hören, dass Subjekt und Prädikat im Deutschen in der Kategorie Numerus kongruieren. Aufmerksamkeit können dagegen Kongruenz-Zweifelsfälle wecken, die beim Entwurf einer Grammatik zunächst sinnvollerweise außen vor bleiben: Eine Reihe schwarze Schafe standen oder stand auf dem Weg? 
„Schwankung“ und „Variation“ das damit verbundene praktische (Orientierungs-) Problem sozusagen aus der Welt theoretisiert werden. Gerade die eingangs thematisierte, sprachpraktische Laienperspektive, die im Begriff des Zweifelsfalls noch unmittelbar präsent ist (,ein x zweifelt über zwei bestimmte sprachliche Formen"), wird in der deskriptiv-analytischen wissenschaftlichen Arbeit, die nicht beratend und für Laien orientierend angelegt ist, ${ }^{8}$ also immer wieder - bewusst oder unbewusst - in den Hintergrund gedrängt. ${ }^{9}$

Angesichts der gerade beschriebenen wissenschaftshistorischen und sprachtheoretischen Vorgaben und Vorarbeiten ist es nicht erstaunlich, dass zur simplen Frage, welche sprachlichen Zweifelsfälle es eigentlich in der deutschen Gegenwartssprache gibt, bis jetzt keine detaillierten empirischen, methodisch kontrollierten sprachwissenschaftlichen Untersuchungen durchgeführt wurden. ${ }^{10}$ Diesbezüglich ist insbesondere daran zu erinnern, dass bestimmte Faktoren mit dem Auftreten und dem Profil der sprachlichen Zweifelsfälle korrelieren könnten. Ich nenne als Illustration nur die folgenden beispielhaften Punkte:

Region: Gibt es eine unterschiedliche Verteilung der sprachlichen Zweifelsfälle in Nord- und Süd-, Ost- und West-Deutschland? Gehen darin möglicherweise noch die älteren Dialektgrenzen mit ein?

Alter: Zweifeln jüngere Sprecher über andere Fälle als ältere? Wenn ja, welche Klassen lassen sich bilden und wie kann man die Verteilung erklären?

Geschlecht: Zweifeln männliche Sprecher tendenziell über andere Fälle als weibliche? Wenn ja, welchen Klassen lassen sich bilden und wie kann man die Verteilung erklären?

Bildung: Wie wirken sich unterschiedliche Bildungsgrade auf die Existenz und den Umgang mit sprachlichen Zweifelfällen aus?

Kommunikationssituation: Welche Auswirkungen besitzen unterschiedliche Kommunikationssituationen (z. B. mündlich/schriftlich, Nähe-/Distanz-Situation) auf die Bewusstwerdung sprachlicher Zweifelsfälle?

Zeit (Wandel): Wie hat sich die Existenz der Zweifelsfälle und der Umgang mit ihnen in den letzten Jahrhunderten der deutschen Sprachgeschichte verändert?

So defizitär die Lage auch ist, so lassen sich doch zumindest einige provisorische, empirisch einigermaßen gestützte Zugänge zur Existenz der gegen-

8 Zur Problematik der Unterscheidung von deskriptiver und präskriptiv-normativer Sprachbetrachtung vgl. Klein 2004a.

9 Insofern stellen Arbeiten wie die von Antos 1996 oder Linke/Ortner/Portmann-Tselikas (Hg.) 2003 (leider) eher seltene Ausnahmen dar.

$10 \mathrm{Vgl}$ zu möglichen Untersuchungsperspektiven auch Klein 2006. 
wärtigen Zweifelsfälle finden. Sie stammen überwiegend aus dem Umfeld von Institutionen, zu deren Kerngeschäft es gehört, sprachliche Zweifelsfälle $\mathrm{zu}$ thematisieren, nämlich Sprachberatungsstellen. Es gibt beispielsweise Arbeiten, in denen erste Ansätze zu einer systematischen Aufarbeitung der Zweifelsfälle formuliert wurden. ${ }^{11}$ Sie verbleiben am Ende allerdings häufig im Impressionistischen und Ungefähren. Die oben genannten Faktoren greifen sie jedenfalls nicht nachdrücklich und systematisch auf. Daneben existieren Variantenwörterbücher, deren Lemmabestand wenigstens als potentielles Reservoir für die Existenz von sprachlichen Zweifelsfällen interpretiert werden kann. ${ }^{12}$

Als Königsweg zur Beantwortung der Frage, welche Zweifelsfälle es in der deutschen Gegenwartssprache gibt, müssen jedoch die Nachschlagewerke genannt werden, die man als Zweifelsfallsammlungen bezeichnen kann. ${ }^{13}$ Die Einführungen dieser Werke geben an, dass das hier registrierte Sprachmaterial aus der konkreten Arbeit der Sprachberatungen hervorgegangen ist. ${ }^{14} \mathrm{Mit}$ anderen Worten, in den Zweifelsfallsammlungen sind diejenigen Fragen bzw. Fragenkomplexe verzeichnet, die (häufig) in den verschiedenen Dienstleistungszusammenhängen der Sprachberatungsstellen zum Gegenstand gemacht werden. Sie repräsentieren insofern die sprachlichen Zweifelsfälle der deutschen Gegenwartssprache. Zu diesen gedruckten Zweifelfallsammlungen kamen zuletzt auch noch entsprechende Internet-Sammlungen hinzu, die ein ähnliches, wenn auch weniger offizielles, institutionell abgesichertes Profil besitzen. ${ }^{15}$

Auch wenn diese Zweifelsfallsammlungen durchaus umfangreich und detailliert sind, so sollte man sich doch klar machen, dass ihre empirische Aussagekraft angesichts der hier diskutierten Frage (welche Zweifelsfälle gibt es in der deutschen Gegenwartssprache?) nicht unproblematisch ist. Das gilt in ganz unterschiedlichen Dimensionen. Zunächst sticht der partielle Charakter der dokumentierten Zweifelsfälle ins Auge. Denn es werden nur diejenigen Fälle behandelt, die Gegenstand einer telefonischen oder schriftlichen Anfrage geworden sind. Faktisch wird das der Spitze eines Eisbergs gleichkommen, weil die Sprecher, die mit Zweifelsfällen konfrontiert sind, nur in den seltensten Fällen die Dienste einer Sprachberatung konsultieren werden. Es

11 Vgl. z. B. Kolde 1976, ders. 1980, Höhne 1990, Mackowiak/Steffen 1991, Scholze-Stubenrecht 1991, Cölfen 1996, Kühn/Almstädt 1997, Müller 1998, Frilling 2004, 2005, Wolf-Bleifuß 2004.

12 Vgl.z.B. Muthmann 1994, Ammon u.a. 2004.

13 Vgl. Duden 2007, Wahrig 2003, für die ehemalige DDR Dückert/Kempcke 1989.

14 Vgl. beispielhaft: ,Der Dudenband ,Richtiges und gutes Deutsch' ist aus der täglichen Arbeit der Dudenredaktion heraus entstanden. Er gibt [...] Antwort auf Fragen, wie sie der Duden-Sprachberatung täglich am Telefon gestellt werden." (Duden 2007, Vorwort).

15 Vgl. z.B. die Internet-Präsentation „Deutsche Sprache - Fragen und Antworten“ (http://faql.de/) oder den Abschnitt „Deutsche Sprache“ unter http://www.wer-weisswas.de. 
gibt also sicher noch sehr viel mehr Zweifelsfälle bzw. Zweifelsfallsituationen als die, die in den Zweifelsfallsammlungen erscheinen.

Aus dem Umstand, dass der primäre Zweck der Zweifelsfallsammlungen nicht in der Dokumentation der Anfragen, also der Zweifelsfälle, sondern in deren Beantwortung liegt, folgt eine weitere Beschränkung der empirischen Reichweite. Denn es ist nicht in allen Fällen klar, welcher konkrete Zweifelsfall eigentlich Anlass für die jeweilige lexikographische Information gegeben haben könnte und wie er im Beratungsgespräch konkret formuliert wurde. Der Darstellung des Wortpaars vergeblich/vergebens ${ }^{16}$ könnten beispielsweise mindestens drei unterschiedliche Fragestellungen zugrunde liegen: Gibt es einen Bedeutungsunterschied zwischen den beiden Wörtern (= semantischer Zweifelsfall)? Kann man diese beiden Wörter in allen Satzkontexten gleichermaßen gebrauchen (= syntaktischer Zweifelsfall)? Folgen beide Wörter den Wortbildungs- bzw. Derivationsregularitäten der deutschen Gegenwartssprache (= morphologischer Zweifelsfall)?

Besonders problematisch für die empirische Stichhaltigkeit der Zweifelsfallssammlungen ist allerdings deren Verankerung in einer vergleichsweise alten, reichen Textsortentradition. Spätestens seit dem 19. Jahrhundert existieren nämlich für den deutschen Sprachbereich entsprechende Sammlungen. ${ }^{17}$ Unabhängig davon, dass sie nicht an allen Punkten von derselben Stoßrichtung geprägt sind, konstituieren sie einen - mehr oder weniger homogenen Überlieferungszusammenhang. ${ }^{18}$ Wie in lexiko- und grammatikographischen Kontexten üblich, muss insofern damit gerechnet werden, dass entsprechende Publikationen von Fall zu Fall eher an bereits existierende Sammlungen anknüpfen, als tatsächlich eigenständige, aktuelle Empirie zu betreiben. Am Rande sei bemerkt, dass die Zweifelsfallsammlungen in dieser Sicht Bestandteile sprachdidaktisch-sprachkritischer Traditionen darstellen, die möglicherweise zur andauernden Existenz von Situationen des Sprachzweifels beitragen. ${ }^{19}$

Was die bisherige linguistische Untersuchung der sprachlichen Zweifelsfälle angeht, sei also resümiert, dass sie aus ganz verschiedenen Gründen noch vergleichsweise wenig analysiert wurden. Dieses Defizit erscheint besonders dann in einem deutlichen Licht, wenn man daran denkt, dass sie nicht nur am

16 Vgl. Duden 2007, s. v. ,,vergebens/vergeblich“, Dückert/Kempcke 1989, s. v. „, vergebens“, ,vergeblich".

17 Ich nenne hier in illustrierender Absicht nur Sanders 1872, Wustmann 1891, Matthias 1892, Engel 1918, dazu etwa Meyer 1993.

18 Zur diesbezüglichen Geschichte vor dem 19. Jahrhundert vgl. Siebenborn 1976, Cherubim 2001, Cherubim/Walsdorf 2004, nach dem 18. Jahrhundert v.a. Cherubim 1983.

19 Vgl. insgesamt zur Wirkung dieser Traditionen Davies/Langer 2006. Als Angelpunkt vieler gegenwärtiger Auffassungen müssen vor allem die drastischen Formen der Sprachkultivierung und Sprachbewertung des 19.Jahrhunderts gelten, die sozialgeschichtlich mit der Etablierung des deutschen Nationalstaats sowie der deutschen Standardsprache verbunden sind, vgl. Dieckmann (Hg.) 1989, Linke 1991, dies. 1996, Lühr 1992, Schrodt 1995. 
Rande linguistischer Arbeiten erwähnt werden sollten, sondern einen genuinen Gegenstand der Sprachwissenschaft darstellen könnten. Für eine adäquate Untersuchung wäre mindestens eine Integration systemlinguistischer und soziolinguistischer Zugänge nötig, um sprachliche Zweifelsfälle angemessen als konstitutiven Bestandteil einer Sprache zu konzeptualisieren und entsprechend empirisch zu erforschen.

Bevor nun die sprachlichen Zweifelsfälle im Zusammenhang der Normierungsproblematik behandelt werden sollen, sei vorab noch eine Unterscheidung grundlegender Typen diskutiert. Dabei werde ich mich im Kern auf die kursierenden Zweifelsfallsammlungen stützen, versuche allerdings Fälle herauszugreifen, die angesichts der oben genannten Definition relativ eindeutig Zweifelsfälle der deutschen Gegenwartssprache darstellen dürften. Nimmt man die Varianten eines Zweifelsfalls in den Blick, so lässt sich eine Klassifikation erstellen, bei der der Sprachgebrauch als ordnender Faktor fungiert. Je nach Verteilung der Varianten im Usus kann man zunächst verschiedene Formen von konditionierten Zweifelsfällen von den unkonditionierten unterscheiden. Als konditioniert möchte ich diejenigen Zweifelsfälle bezeichnen, deren Varianten zumindest teilweise in unterschiedlichen Kontexten verankert sind und die man daher nicht in allen Fällen gegeneinander austauschen kann. Anders gesagt: bei diesen Zweifelsfällen lassen sich Bedingungen benennen, die den Gebrauch der Varianten steuern. Ihr Usus ist weitgehend disjunktiv auf unterschiedliche Kommunikationssituationen abbildbar. Oft gilt also: Variante A kann nur in Situationen des Typs A' verwendet werden, Variante B nur in Situationen des Typs B'. Wer Variante A (B) in Situationen des Typs B' (A') nutzt, produziert fehlerhafte Sprache.

Als Unterklassen der konditionierten Zweifelsfälle können dann Zweifelsfälle differenziert werden, deren Gebrauch morphosyntaktisch, semantisch, pragmatisch oder durch Sprachwandel determiniert ist. Bei den pragmatisch bedingten Fällen können wiederum Subklassen unterschieden werden, je nachdem welcher pragmatische Faktor die Verteilung der Varianten bedingt. $\mathrm{Zu}$ nennen sind vor allem die mediale Dimension (z. B. schriftliche vs. mündliche Sprache), die regionale Verteilung (z. B. süddeutsche vs. norddeutsche Sprachformen) sowie funktionale Faktoren (z. B. Fachsprache vs. Standardsprache). ${ }^{20}$

20 In Anbetracht der Spannbreite pragmatischer Faktoren können hier von Fall zu Fall auch noch andere Motive wirken, so beispielsweise stilistische und textsortenbezogene Faktoren oder (situative) Dimensionen wie Nähe vs. Distanz, höflich vs. unhöflich sowie das Geschlecht und das Alter. Mit den oben aufgeführten pragmatischen Bedingungen lässt sich aber vermutlich der größte Teil der pragmatisch konditionierten Zweifelsfälle analysieren. Nicht selten spielen auch mehrere pragmatische Faktoren gleichzeitig eine gewisse Rolle, vgl. am Beispiel des Zweifelsfalls siebte/siebente etwa Bellmann 2002. 
Bei unkonditionierten Zweifelsfällen ist demgegenüber die (klare) Identifikation derartiger Steuerungsfaktoren (noch) nicht möglich; ihre Verteilung im Usus erscheint sozusagen zufällig, ohne dass man angeben könnte, warum im einen Fall die Variante A, im anderen Fall die Variante B genutzt wird. Unkonditionierte Zweifelsfälle werden also durch Varianten repräsentiert, deren Gebrauch tatsächlich schwankt und die daher auch Zweifelsfälle im engeren Sinn oder auch echte Zweifelsfälle genannt werden könnten. Zur Illustration seien kurz einige beispielhafte Fälle in einer Übersicht angeführt:

\begin{tabular}{|c|c|c|c|}
\hline \multicolumn{3}{|c|}{ Konditionierte Zweifelsfälle } & Unkonditionierte \\
\hline & & \multicolumn{2}{|l|}{ Beispiele } \\
\hline \multicolumn{2}{|c|}{ 1. Morphosyntaktisch } & vergeblich/vergebens ${ }^{21}$ & \multirow{3}{*}{$\begin{array}{l}\text { kargerlkärger } \\
\text { MorastelMoräste } \\
\text { irgendwelches dummes } \\
\text { Zenglirgendwelches } \\
\text { dumme Zeug } 22\end{array}$} \\
\hline 2. Semantisch & & DrachelDrachen, wog/wiegte, & \\
\hline \multirow[t]{4}{*}{ 3. Pragmatisch } & a. medial & $\begin{array}{l}\text { bekommen/kriegen, } \\
\text { werfen/schmeißen }\end{array}$ & \\
\hline & b. regional & $\begin{array}{l}\text { hat/ist gesessen, } \\
\text { benutzen/beniutzen }\end{array}$ & \multirow{4}{*}{$\begin{array}{l}\text { Zwei und zwei ist/ } \\
\text { sind vier. }\end{array}$} \\
\hline & c. funktional & lackenllackieren & \\
\hline & d. andere... & $\begin{array}{l}\text { [z. B. stilistisch, situativ, } \\
\text { textsortenabhängig...] }\end{array}$ & \\
\hline \multicolumn{2}{|l|}{ 4. Sprachwandel } & $\begin{array}{l}\text { eine Herde schwarzer Schafel } \\
\text { eine Herde schwarze Schafe }{ }^{23} \text {, } \\
\text { GlaubelGlauben }{ }^{24} \text {, } \\
\text { dem Helden/dem Held }{ }^{25}\end{array}$ & \\
\hline
\end{tabular}

Tab. 1: Typen sprachlicher Zweifelsfälle nach Sprachgebrauch

${ }^{21} \mathrm{Vgl}$. generell zu dieser Problematik, in der sich die grammatisch keineswegs triviale Abgrenzung zwischen Adjektiven und Adverbien verkörpert, Eisenberg $2002 \mathrm{a}$.

$22 \mathrm{Vgl}$. zu dieser Flexionsproblematik den Aufsatz von Bernd Wiese in diesem Band.

23 Vgl. zu dieser Entwicklung z. B. Glaser 1992.

24 Vgl. zu dieser Entwicklung z. B. Joeres 1996.

25 Vgl. zu dieser Entwicklung z. B. Thieroff 2003. 
Der Steuerungsgehalt der verschiedenen Faktoren lässt sich bei den konditionierten Zweifelsfällen dann in der folgenden Art und Weise systematisieren:

\begin{tabular}{|c|c|c|}
\hline \multirow{2}{*}{$\begin{array}{l}\text { Morpho- } \\
\text { syntaktisch }\end{array}$} & attributiv/adverbial/prädikativ & adverbial/prädikativ \\
\hline & vergeblich & vergebens \\
\hline \multirow[t]{2}{*}{ Semantisch } & Bedeutung 1 & Bedeutung 2 \\
\hline & $\begin{array}{l}\text { Drache ,Fabelwesen“ } \\
\text { wog, Gewicht bestimmen“ }\end{array}$ & $\begin{array}{l}\text { Drachen ,Kinderspielzeug" } \\
\text { wiegte, hin und her bewegen }\end{array}$ \\
\hline \multirow[t]{6}{*}{ Pragmatisch } & medial-schriftlich & medial-mündlich \\
\hline & bekommen, werfen & kriegen, schmeißen \\
\hline & regional/norddt. & regional/süddt. \\
\hline & hat gesessen, benutzen & ist gesessen, benützen \\
\hline & funktional-fachsprachlich & funktional-standardspr. \\
\hline & lacken & lackieren \\
\hline \multirow[t]{2}{*}{ Sprachwandel } & ältere Sprachform & neuere Sprachform \\
\hline & $\begin{array}{l}\text { eine Herde schwarzer Schafe } \\
\text { Glaube } \\
\text { dem Helden }\end{array}$ & $\begin{array}{l}\text { eine Herde schwarze Schafe } \\
\text { Glauben } \\
\text { dem Held }\end{array}$ \\
\hline
\end{tabular}

Tab. 2: Konditionierte Zweifelsfälle: Beispiele für Steuerungstypen

Vor dem Hintergrund der obigen Klassifikation (Tabellen 1,2) lässt sich nun die Frage der Normierung der Zweifelsfälle erörtern. Dazu sind zunächst einige grundsätzliche Vorbemerkungen nötig. Sie beziehen sich auf die Art und Weise sowie die Ziele, die bei dieser Normierung anzusetzen sind.

Sprachliche Normierungsprozesse vollziehen sich oft als explizite Setzungen normativer Aussagen. ${ }^{26}$ Durch sie wird festgelegt, wie man bestimmte Wörter oder Konstruktionen gebrauchen sollte. Im Kontext der Zweifelsfälle ergeben sich dafür spezifische Rahmenbedingungen. Denn explizit festgelegte Sprachnormen sind hier prinzipiell als erwünschte Beratung der Sprecher zu konzeptualisierten. Sie erscheinen als Informationen, durch die bestimmte sprachlich-kommunikative Desorientierungszustände (,Zweifelsfallsituationen“) bewältigt werden können. Ihr Zweck liegt demnach darin, dass sie für ganz konkrete Situationen Handlungsanweisungen darstellen. Sie sind für die

26 Natürlich existiert durch den herrschenden Sprachgebrauch immer auch eine implizite Normierung, die nicht explizit festgehalten werden muss und trotzdem eine nicht unerhebliche normative Kraft besitzen kann. Begrifflich lassen sich in diesem Sinn explizite Normen von Gebrauchsnormen unterscheiden. Mein Begriff von Sprachnormierung wird sich im folgenden aber ausschließlich auf die explizite Normierung der Sprache beziehen, die im Deutschen in den verschiedensten metasprachlichen Texten erfolgt. Auch die Sprachberatung am Telefon oder per E-Mail kann in diesem Sinn als Form der expliziten Sprachnormierung gesehen werden. 
Sprecher insofern relevant, als sie im Moment sprachlich nicht handeln können, aber durchaus handeln wollen.

Diese etwas ungelenke Beschreibung soll auch klar machen, wofür Sprachnormen in diesem Zusammenhang gerade nicht zu entwerfen sind. Es geht nämlich keinesfalls darum, dass man mit ihrer Hilfe in alter präskriptiver Manier im Sprachgebrauch der Anderen „Fehler“ identifizieren möchte. Das würde sozusagen einer ,unerwünschten Beratung“ entsprechen, die bei Zweifelsfällen eben gar nicht zur Debatte steht. Sprachnormierung im Kontext der Zweifelsfälle ist vielmehr eine Art Hilfestellung in einem Entscheidungsprozess, bei dem die Sprecher unter Handlungsdruck stehen. Allerdings zeigt die Geschichte der Sprachnormierungen, dass derartige Beratungsprozesse nicht selten in das Raisonnieren über Sprachfehler umschlagen können. ${ }^{27}$ Wer die Beratungsperspektive generalisiert, ohne dass im Einzelfall tatsächlich Beratung von den Sprechern erwünscht ist, neigt dazu, im beobachtbaren Sprachgebrauch „Fehler" zu identifizieren. Es sei daher ausdrücklich vermerkt, dass die folgenden Ausführungen nicht in diesem Sinn verstanden werden sollten. Im Zentrum steht ein Beratungs-, kein Fehlerdiskurs.

Ein solcher Beratungsdiskurs muss das Ziel haben, einen rationalen Umgang mit Sprachproblemen zu etablieren. ${ }^{28}$ Insbesondere ist anzustreben, die Entscheidungsgrundlagen für die Zweifelnden transparent und nachvollziehbar zu machen. Damit ist in letzter Konsequenz auch das Ziel verbunden, dass die Ratsuchenden viele Zweifelsfälle tendenziell aufgrund eigener Überlegungen, Erfahrungen und Kenntnisse selbständig klären können, sofern ihnen nur die prinzipiellen Entscheidungsprozeduren deutlich (gemacht worden) sind. Ein rationaler Umgang mit Sprachproblemen besteht insofern darin, die Ratsuchenden soweit wie möglich als Subjekte von Entscheidungen einzusetzen und sie nicht als bloße Rezipienten normativer Feststellungen anzusehen. Gerade in der Selbstreflexion sollten sie sich in sprachlichen Zweifelsfällen nicht einfach als Befehlsempfänger sprachlicher Normen begreifen. ${ }^{29}$

Um es noch einmal deutlich zu formulieren: Ich gehe davon aus, dass die zweifelnden Sprecher in vielen (nicht in allen!) Fällen bereits dunkel und unbegriffen über das Sprachwissen zur Klärung eines Zweifelsfalls verfügen. $\mathrm{Zu}$ verweisen ist in dieser Hinsicht einerseits auf das jedem (muttersprachlichen) Sprecher zugängliche Sprachgefühl, andererseits auf seine (quasi-

27 Entsprechende Entwicklungen sind in der Forschung bisher leider kaum untersucht worden; zu ersten Überlegungen und Vorarbeiten auf diesem Feld vgl. Andersson/Trudgill 1990, Konopka 1996, Davies/Langer 2006, Klein 2003.

29 Selbstverständlich bin ich mir der Tatsache bewusst, dass den genannten Zielen angesichts der herrschenden Praxis und des teilweise verstörend irrationalen Laiensprachbewusstseins ein gewisses utopisches Moment anhaftet. Auf lange Sicht und insbesondere unter sprachdidaktischen Perspektiven halte ich es jedoch nicht für völlig unrealistisch, einer rationalen Reflexions- bzw. Diskussionskultur und Beratungspraxis in sprachlichen Dingen immer näher zu kommen. 
empirischen) Kenntnisse des realen Sprachgebrauchs. In einem rationalen Beratungsdiskurs wäre insofern nur danach zu streben, diese bereits vorhandenen Sprachkenntnisse mit den notwendigen Klärungs- bzw. Entscheidungsstrategien zu verbinden. Dadurch würden die Sprecher auch nachhaltig lernen, dass sie Zweifelsfälle oft auch ohne Konsultation normativer Texte durchaus angemessen und vernünftig in den Griff bekommen können. Nicht zuletzt wären sie mit der Tatsache konfrontiert, dass in jedem Sprachgebrauch ohnehin Freiheiten herrschen, die sich die Sprecher, bewusst oder unbewusst, nehmen können, ohne damit gegen irgendwelche angeblichen „Gesetze“ zu verstoßen.

Zweifelsfälle erscheinen vor diesem Hintergrund tendenziell als sprachliche Probleme, die durch eigene, nachvollziehbare Anstrengungen und nicht durch die bloße „Befolgung“ präskriptiver, autoritativer Normtexte bewältigt werden können. Für ein solches Vorgehen spricht nicht zuletzt - um hier einmal einen großen Horizont aufscheinen zu lassen - unsere sozial- und kulturgeschichtliche Gegenwart. Denn die deutsche Sprache ist am Anfang des 21. Jahrhunderts als demokratisches Gemeingut zu fassen, nicht mehr wie früher als elitäre Gruppensprache des (Bildungs-)Bürgertums. Dementsprechend sollte sie auch nicht mehr mit den hochgradig sprachautoritären, sozial differenzierenden Mechanismen und Verfahren des 19. Jahrhunderts reflektiert werden, sondern im Stil einer demokratisch-rationalen Verfassung, die gerade nicht auf Befolgung von Autoritäten und unreflektierter Normrezeption basiert. ${ }^{30}$

Als Bezugsbasis für die Klärung der Zweifelsfälle setze ich - wie oben schon angedeutet wurde - die Kenntnis des realen Usus und seiner Regularitäten an. Mit anderen Worten, die Analyse der Zweifelsfälle erfolgt auf der Basis der herrschenden Gebrauchsnormen, die den Usus der deutschen Gegenwartssprache bestimmen. ${ }^{31}$ Insbesondere mit Blick auf die öffentliche Sprachdiskussion muss klar sein (bzw. je nach Bedarf klar gemacht werden können), dass die Logik der Sprachberatung in Zweifelsfällen weder auf Buchautoritäten (z. B. Duden, Wahrig ...) noch institutionellen Autoritäten (z. B. Sprachakademien, Sprachgesellschaften), Personenautoritäten (z. B. Grammatiker, Schriftsteller, Lehrer, Professoren, Bastian Sick ...) oder Sprachsystemautoritäten (z. B. (abstrakte) Sprachregeln, Grammatiktheorien) beruht. Aus-

30 Nicht wenige Haltungen und Einstellungen, die in der Sprachöffentlichkeit explizit oder implizit artikuliert werden, wurzeln letztlich immer noch in den hoch gradig sozialsymbolisch aufgeladenen Diskussionen des 19. Jahrhunderts, vgl. für diese Vorgaben der älteren Sprachdiskussion insbesondere Mattheier 1991, Linke 1991, dies. 1996. Der große Erfolg der Sprachratgeber von Bastian Sick muss vor diesem Hintergrund leider als großer Rückschritt in ältere, eigentlich zu überwindende Formen der Sprachbewertung und Sprachthematisierung gesehen werden, vgl. dazu, allerdings mit unterschiedlichen Akzenten, etwa Schneider 2005, Stenschke 2007, Maitz/Elspaß 2007, Ágel 2008, Meinunger 2008.

31 Vgl. dazu auch den instruktiven Bericht über die jüngste Umarbeitung einer wichtigen Zweifelsfallsammlung in Eisenberg 2007. 
schlaggebend ist vielmehr der beobachtbare Sprachgebrauch, insbesondere derjenige, der sich in standardsprachlichen ${ }^{32}$ Kommunikationskontexten verkörpert. Darüber hinaus kann auf dieser Grundlage und angesichts der oben vorgestellten Klassifikation der Zweifelsfälle ein systematisches Entscheidungsverfahren entwickelt werden, um in diesen Fällen angemessen und eindeutig beraten zu können. Ein solches Verfahren berücksichtigt den Umstand, dass Sprecher in manchen Zweifelsfallsituationen sicher keine Wahl haben, sondern sich nur für eine der betreffenden Varianten entscheiden dürfen. In anderen Fällen jedoch bestehen gewisse Wahlmöglichkeiten, die im Zuge der Klärungsprozedur bewusst gemacht werden sollten. Ein solcher Prozess ist durch eine folgerichtige Abarbeitung von jalnein-Entscheidungsfragen zu erreichen; er sieht in der Übersicht wie folgt aus:

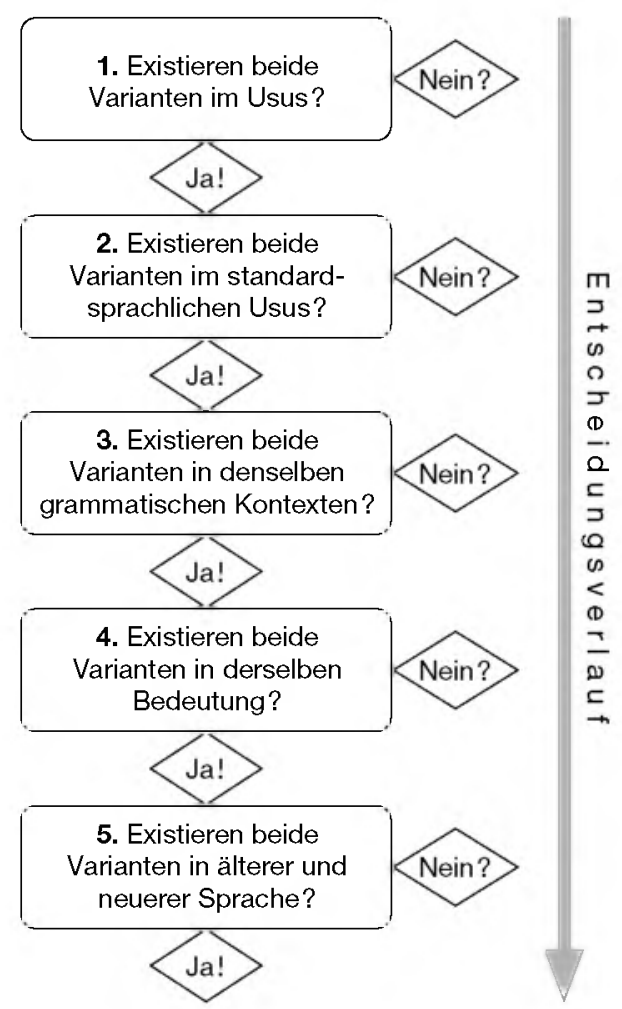

Tab. 3: Zur Klärung der Zweifelsfälle: Entscheidungssystematik

32 Ich gehe also davon aus, dass es möglich ist, einen empirisch legitimierbaren Begriff von Standardsprache zu entwickeln, der einer Entscheidung in Zweifelsfällen zugrunde gelegt werden kann. Als Angriffspunkt für entsprechende Erhebungen wären überregionale, schriftsprachnahe Sprachformen zugrunde zu legen, wie sie sich, schriftlich oder mündlich. beispielsweise in überregionalen Nachrichtensendungen. Zeitungen. Zeitschriften und offiziellen Internet-Seiten finden. 
Bevor die einzelnen Schritte dieser Klärungsprozedur abschließend mit einigen Beispielen etwas genauer vorgestellt werden sollen, möchte ich zur Vermeidung von Missverständnissen noch einige Bemerkungen vorausschicken, die den Status dieser Entscheidungssystematik für die Klärung der Zweifelsfälle betreffen. Aus vielen Arbeitsberichten ${ }^{33}$ von Sprachberatungsstellen ist bekannt, dass die Auskunftsuchenden oft nicht an den Hintergründen und Verfahren für die Entscheidungsfindung interessiert sind. Nicht selten will man als Zweifelnder lediglich wissen, was „richtig" oder „falsch" ist, ohne dass eine Begründung für diese Etikettierung gefragt wäre. Eine solche Bewusstseinslage ist natürlich als Faktum erst einmal zu akzeptieren, auch wenn dadurch die Etablierung einer rationalen, argumentativ angelegten Sprachberatung nicht gerade erleichtert wird. Angesichts dieser Vorgabe sei daher nachdrücklich darauf verwiesen, dass die folgenden Entscheidungsprozeduren keinesfalls immer im 1:1-Verhältnis in Sprachberatungssituationen zu übertragen sind.

Wie in solchen Konsultationskontexten von der Seite der Sprachratgeber, schriftlich oder mündlich, konkret gehandelt werden sollte, bedarf einer eigenen Untersuchung. Die folgenden Ausführungen dürfen also nicht als unmittelbare Handlungsanweisungen für die konkrete dialogische Ausgestaltung der Sprachberatungspraxis missverstanden werden. Sie machen nämlich nur die Entscheidungslogik der Sprachnormierung in Zweifellsfällen explizit, die in vielen Fällen bereits so oder ähnlich praktiziert wird. Insofern richten sich die folgenden Handlungsanweisungen auch eher an diejenigen Personen, die Zweifelsfälle zum Zwecke der Konsultation analysieren und aufbereiten möchten, als an diejenigen, die von den Zweifelsfällen geplagt werden. $\mathrm{Ob}$ und, wenn ja, in welchen Formen entsprechende Entscheidungsprozesse direkt an die Ratsuchenden herangetragen werden, ist zunächst eine Sache der Erfahrung und insbesondere der individuellen Anfrage. Manchmal kann es sinnvoll sein, den Auskunftsuchenden mit einer entsprechenden (oder einer ähnlichen) Frage wie in Tabelle 3 zu konfrontieren, manchmal sollte man sich tunlichst davor hüten. Freilich ist durchaus vorstellbar, dass in anderen $\mathrm{Zu}$ sammenhängen, beispielsweise in sprachdidaktischen Perspektiven (Deutschunterricht!), die Entscheidungssystematik ausdrücklich als solche vorgestellt und besprochen wird.

Ist man mit einem Zweifelsfall, also zwei Varianten, konfrontiert, sind der Reihe nach (höchstens) fünf Schritte mit entsprechenden Fragen (siehe Tabelle 3) durchzugehen. In der Fragenreihe werden systematisch die potentiellen Bedingungskontexte in Rechenschaft gezogen, die bei der Konsultation in Zweifelsfällen zum Tragen kommen können. Die Fragen reflektieren also den Umstand, dass bei der Klärung der Zweifelsfälle verschiedene Faktoren eine Rolle spielen können, man aber noch nicht weiß, welcher Faktor auf welche Art und Weise im Einzelfall der entscheidende ist.

$33 \mathrm{Vgl}$. die Literaturangaben in Fußnote 11. 
Die generelle Logik der Entscheidungsprozedur sieht folgendermaßen aus: Wenn als Antwort ein Ja festgestellt wird, muss die nächste Frage thematisiert werden, um zu einer Klärung zu gelangen. Wird die Ausgangsfrage negiert, ergibt sich auf dieser Ebene bereits eine substantielle Klärung des Zweifelsfalls, die in einer entsprechenden Handlungsanweisung resultiert. Bei vielen Zweifelsfällen werden also nicht sämtliche Fragen durchgegangen, sondern sie lassen sich bereits nach zwei oder drei Fragen aufschlüsseln. In manchen Fällen muss der Entscheidungsprozess allerdings bis zum Schritt 5 abgearbeitet werden, damit sich eine angemessene Beratung ergeben kann.

SCHRITT 1: Zunächst stellt sich das Problem, ob die fraglichen Varianten im Usus des Deutschen überhaupt irgendwo existieren. Bei Verneinung dieser Frage kann man dann im wesentlichen zwei Fälle unterscheiden: a. beide Varianten existieren nicht im Usus, b. eine Variante existiert nicht im Usus, die andere schon. Fall a dürfte einen gewissen hypothetisch-theoretischen Charakter haben, da er kaum vorkommen wird. Man zweifelt höchstwahrscheinlich nicht gleichzeitig über zwei Varianten, mit denen man noch nie irgendwo konfrontiert wurde. Allerdings sollte man die sprachliche Kreativität bei der Wort- und Wortformenbildung nicht unterschätzen. Auch wenn diesbezüglich eine restringierte Beratung sicher nicht unproblematisch ist, ${ }^{34}$ dürfte es der standardsprachliche Zielpunkt der meisten Zweifelsfallsituationen nahe legen, dass eher nach existierenden Formen oder Mustern zu suchen ist, anstatt innovativ-exotische Bildungen zu übernehmen. In diesem Fall würde die Handlungsanweisung also lauten: „1.a.: Suche nach existierenden Varianten und/oder prüfe, ob $\mathrm{Du}$ an bestehende sprachliche Formulierungsmuster anknüpfst!“”.

Im Fall b stellt sich die Sache dagegen erst einmal relativ einfach dar, insofern die im Usus existierende Variante vorzuziehen ist (z. B. der Demut statt die Demut, Dunkelmänner statt Dunkelleute). Aber auch hier sollte man wie bei 1.a. daran denken, dass die sprachliche Kreativität bisher nicht-existierende Formen hervorbringen kann, die nicht einfach als falsch oder unpassend zu klassifizieren sind. Die Entscheidung kann also durchaus vom Einzelfall abhängen. Als Generallinie ist jedoch, wiederum vor allem mit Blick auf die etablierte Standardsprache, festzuhalten „1.b.: Nutze die existierende Variante!“

SCHRITT 2: Schritt 1 betrifft generell die Verankerung der Varianten im deutschen Usus und potentielle Formen von Sprachkreativität; in Schritt 2 erfolgt eine Evaluation der Varianten im Blick auf deren standardsprachlichen oder nicht-standardsprachlichen Gebrauch. Wird die entsprechende Frage ( "Existieren beide Varianten im standardsprachlichen Usus?") negiert, ist also zumindest eine der beiden Varianten als nicht-standardsprachlich identifiziert

34 Vgl. zur Wortbildung Heringer 1984, Donalies 2003. 
(2.a.). Systematisch gesehen könnte auch der (wohl seltene) Fall (2.b.) eintreten, dass beide Varianten als nicht-standardsprachlich zu kennzeichnen sind. Dies ähnelt den unter Schritt 1 diskutierten Fällen und soll daher hier nicht näher diskutiert werden.

Fall 2.a. dagegen kommt sicher häufig vor. Im Hintergrund stehen hier die verschiedenen pragmatisch konditionierten Zweifelsfälle, die unter anderem aus der Varietätenvielfalt des Deutschen und den je spezifischen Kommunikationsbedingungen resultieren (vgl. Tabelle 1,2 „pragmatisch konditionierte Zweifelsfälle“). Meistens wird dabei zur standardsprachlichen Variante zu raten sein. Prinzipiell sollte aber auch klar sein, dass je nach individuellem Fall auch die Nutzung einer anderen Varietät als der standardsprachlichen sinnvoll sein könnte. Hier ist die Sensibilität und Erfahrung derjenigen Personen gefragt, die im betreffenden Fall eine Auskunft geben wollen. Als Handlungsanweisung soll insofern nicht einfach die Bevorzugung der Standardsprache herausgehoben werden, sondern die passende Auswahl je nach Kommunikationskontext: „2.a.: Nutze die Variante derjenigen Varietät, in der Du kommunizieren möchtest!". Handelt es sich bei dem avisierten Kontext um eine Kommunikationssituation, die als standardsprachlich zu charakterisieren ist, ist folglich einfach die standardsprachliche Variante zu selektieren. Bei einem Kommunikationskontext mit nähesprachlich-dialektalem Charakter kann entsprechend die dialektale bzw. regionalsprachliche Variante gewählt werden.

SCHRITT 3 + 4: Wenn die Fragen 1 und 2 bejaht wurden, tauchen für die Schritte 3 bis 5 nur noch Varianten auf, die alle im standardsprachlichen Usus vorkommen. Zur weiteren Klärung müssen nun also Kriterien angesetzt werden, die die morphosyntaktische (Schritt 3) und die semantische (Schritt 4) Dimension ins Spiel bringen und dadurch eindeutige Entscheidungen nach sich ziehen (vgl. Tabelle 1, 2 „morphosyntaktisch bzw. semantisch konditionierte Zweifelsfälle“).

Bei einer negativen Antwort auf Frage 3 („Existieren beide Varianten in denselben grammatischen Kontexten?“) werden diejenigen Zweifelsfälle identifiziert, die morphosyntaktisch konditioniert sind. Entsprechend muss sich die Handlungsanweisung auf die Beachtung des (grammatischen) Kontexts beziehen, damit eine korrekte Entscheidung gefällt werden kann: „3. Achte auf Deinen Äußerungskontext und nutze die passende Variante!" Ergibt sich durch eine affirmative Antwort auf Frage 3, dass keine morphosyntaktische Konditionierung vorliegt, ist durch Frage 4 („Existieren beide Varianten in derselben Bedeutung?") die Möglichkeit einer semantischen Konditionierung zu erwägen. Wenn dem so ist (= negative Antwort auf Frage 4), verweist die Handlungsanweisung entsprechend auf die gebotene Berücksichtigung der Bedeutungsdimension: „4. Achte auf die Bedeutung Deiner Äußerung und nutze die passende Variante!“ Demgemäß würden an dieser Stelle semantisch konditionierte Variantenpaare geklärt werden. 
SCHRITT 5: Sollte der Zweifelsfall nach den ersten vier Fragen noch nicht geklärt sein, handelt es sich also um ein Paar, dessen Varianten gleichermaßen in der Standardsprache vorkommen, die aber nicht an unterschiedliche morphosyntaktische Kontexte gebunden sind und die auch keine unterschiedlichen Bedeutungen transportieren. An dieser Stelle ist nun der Faktor geltend zu machen, dass viele Zweifelsfälle aus Sprachwandelvorgängen resultieren (vgl. Tabelle 1, 2 „durch Sprachwandel konditionierte Zweifelsfälle"). Es kann also gegebenenfalls eine eher ältere und eine eher jüngere Variante geben. Darauf zielt die Frage 5 „Existieren beide Varianten in älterer und neuerer Sprache?" Bei negativer Antwort liegt demnach eine Konditionierung durch Sprachwandel vor und die Handlungsanweisung muss den entsprechenden Faktor explizit thematisieren. Insofern erscheint es sinnvoll, dem Sprecher unmittelbar die Wahl zwischen älterer und neuerer Sprache zu überlassen. Dadurch wird auch klar, dass er mit seiner Auswahl den Sprachwandel entweder vorantreiben oder aufhalten könnte, je nachdem ob er die ältere oder die neuere sprachliche Einheit selektiert. Die Entscheidung über die Kraft einer Entwicklungstendenz liegt beim Sprecher und sollte ihm auch als solche bewusst sein: „5. Entscheide Dich, ob Du eher innovativ den Sprachwandel vorantreiben oder konservativ das Alte bewahren möchtest, und wähle die entsprechende Variante!“

SCHRITT 6: Wenn alle Fragen der Klärungssystematik bejaht worden sind, kommt das dem Umstand gleich, dass man unkonditionierte Zweifelsfälle identifiziert hat (vgl. Tabelle 1 „unkonditionierte Zweifelsfälle“). Es lassen sich also nach dem bisherigen Stand der Dinge keine Faktoren angeben, die den differenzierten Gebrauch erklären könnten und insofern als ansprechbare Steuerungspotentiale verfügbar wären. Aber auch in diesem Fall sollte den Sprechern natürlich eine eindeutige Handlungsanweisung gegeben werden, damit sie den jeweiligen Zweifelsfall als explizit thematisiert und rational behandelt begreifen können. Eine angemessene Behandlung muss hier allerdings die faktische Freiheit der Sprachbenutzer in den Mittelpunkt rücken und darauf aufbauend ein Auswahlverfahren vorschlagen. Vor diesem Hintergrund formuliere ich die Handlungsanweisung „6.: Wähle die Variante, die Deinem Sprachgefühl am besten entspricht!" Es ist hervorzuheben, dass - anders als gelegentlich zu hören ist - diese Empfehlung keiner schwammigen Kapitulation einer ausschließlich deskriptiv arbeitenden Linguistik gleichkommt, sondern systematisch aus dem Charakter des identifizierten Zweifelsfalls folgt: Wo (noch) keine präzis identifizierbaren Rahmenbedingungen herrschen, kann der einzelne Sprecher - wie in vielen anderen Fällen auch - legitimerweise seiner persönlichen sprachlichen Vorliebe folgen.

Die systematische Entscheidungsprozedur für sprachliche Zweifelsfälle gemäß Tabelle 3 ist, wie deutlich geworden sein dürfte, ein gestaffeltes Verfahren, in dem einerseits gewisse Zwänge herrschen, andererseits aber immer wie- 
der Wahlmöglichkeiten offen stehen. Die Systematik der Abfrageprozedur folgt dabei den grundlegenden Vorgaben, in denen die Existenz von Zweifelsfällen angesiedelt ist und die daher für ihre Bewältigung zu berücksichtigen sind: Zunächst ergibt sich eine Betrachtung des Usus in der gesamten deutschen Sprache, dann werden eventuelle Varietätenbindungen sowie gegebenenfalls spezifische morphosyntaktische und semantische Vorgaben der Standardsprache aufgenommen. Zuletzt tauchen eventuelle Sprachwandelvorgänge auf.

Aus diesem Konglomerat ergibt sich ein Gerüst von Handlungsanweisungen, die letztlich die Freiheiten und Notwendigkeiten eines Individuums beim sprachlichen Kommunizieren reflektieren. Eine solche Systematik ist der Natur der Sprache unmittelbar angemessen, da bei jeder Sprachproduktion gewisse soziale Vorgaben zu berücksichtigen sind, gleichzeitig aber immer wieder individuelle, gegebenenfalls auch ganz freie Entscheidungen der kommunizierenden Personen anfallen. In dieser Sicht aktualisiert sich bei der rationalen Bewältigung der Zweifelsfälle nur etwas, was bei jedem sprachlichen Kommunizieren immer wieder geleistet werden muss, nämlich der Umgang mit einem mehr oder weniger komplexen Gefüge von sprachlichen Notwendigkeiten und sprachlichen Freiheiten. Wer Zweifelsfälle im beschriebenen Sinn rational angeht, weitet also nur explizit und bewusst die Strategien und Verfahren aus, die bei jedem sprachlichen Handeln ohnehin implizit befolgt werden.

Auch im Sinne eines Resümees soll angesichts der obigen Darlegungen abschließend die Frage des Titels aufgenommen werden: Inwiefern stellen also Zweifelsfälle Herausforderungen für Sprachwissenschaft und Sprachnormierung dar? Und was steht hier eigentlich auf der Kippe? Auch wenn die Perspektiven sicher nicht immer sauber zu trennen sind, möchte ich meine Antwort auf diese Frage zunächst auf die Sprachwissenschaft, dann auf das Geschäft der Sprachnormierung bzw. Sprachberatung beziehen.

Aus Sicht der Sprachwissenschaft stellen die Zweifelsfälle eine vielschichtige Herausforderung dar, weil damit die Arbeit auf einem Feld angesprochen ist, das traditionell eher marginalisiert wurde. Bei der kontinuierlichen Ermittlung der empirischen Fakten sowie der Formulierung der theoretischen Grundlagen für eine angemessene Sprachbeschreibung der Zweifelsfälle kann also, im Unterschied zu vielen anderen Themen der Sprachwissenschaft, nicht auf etablierte Fragemuster und erprobte Forschungsansätze zurückgegriffen werden. Insbesondere steht die Sprachwissenschaft hier unter dem externen Druck, Standardsprachlichkeit inklusive eventueller Entwicklungstendenzen zu identifizieren. Denn nur wenn man wei $\beta$, was standardsprachlich üblich ist, wird man viele Zweifelsfälle kommunikativ adäquat und einschlägig öffentlichkeitsrelevant klären können. Vor diesem Hintergrund muss notwendigerweise eine umfassende Konzeptualisierung und empirische Identifikation sprachlicher Variation in sämtlichen Dimensionen, d. h. sowohl grammatisch- 
systemlinguistisch als auch pragmatisch-soziolinguistisch, geleistet werden. Auch die sozialsymbolischen Dimensionen von Sprachlichkeit, ohne die die gesellschaftliche Realität der Zweifelsfälle nicht angemessen erfasst werden kann, sind dabei nachdrücklich zu berücksichtigen.

So wäre beispielsweise auf breiter Datengrundlage zu erheben und zu analysieren, wie „Beratungsdiskurse“ in „Fehlerdiskurse“ umschlagen können und wie in diesem Zusammenhang der tatsächliche Status „fehlerhafter“, nicht-varietätenkonformer Sprache zu bestimmen ist. Darüber hinaus sind die Zweifelsfälle möglichst facettenreich sprachhistorisch aufzuarbeiten. Auch hier reicht eine systemlinguistische Sicht nicht aus. Sie muss durch entsprechende pragmatische, variationslinguistische und metasprachlich ausgerichtete Untersuchungen vervollständigt werden. Um nur ein eher bekanntes, aber längst nicht ausdiskutiertes Beispiel auf diesem Feld zu nennen: Können (öffentlichkeitsrelevante, sprachdidaktische, sprachkritische) Normdiskurse die objektsprachliche Entwicklung der Zweifelsfälle beeinflussen und, wenn $\mathrm{ja}$, wie lassen sich die Muster und Bewegungen dieser Beeinflussung beschreiben? ${ }^{35}$ Dazu sollten zum Schluss sprachvergleichende Perspektiven treten, in denen die (objektsprachliche) Existenz und die (metasprachliche) Bewältigung sprachlicher Zweifelsfälle in unterschiedlichen kulturellen und gesellschaftlichen Kontexten analysiert werden. Denn die Art und Weise, wie man mit Zweifelsfällen in Deutschland umgeht, dürfte - zumindest in Teilen nicht mit entsprechenden Strategien und Stilen in anderen Sprachen und gesellschaftlichen Zusammenhängen übereinstimmen.

Sprachnormierung stellt sich bei den Zweifelsfällen, wie oben ausgeführt, als Sprachberatung dar. Demzufolge erhebt sich hier die Frage nach der Erstellung angemessener, adressatengerechter Beratungstexte und Didaktisierungen zu den fraglichen sprachlichen Einheiten - natürlich stets unter Berücksichtigung der linguistisch ermittelten Fakten und Konzeptualisierungen. Im Raum stehen etwa Belange des Deutschunterrichts und von Deutsch als Fremdsprache (DaF). Es ist in diesem Zusammenhang nachdrücklich hervorzuheben, dass die Aufarbeitung der sprachlichen Tatsachen zum Zwecke der Konsultation keineswegs trivial ist. Die Unterstellung, dass man die maßgeblichen linguistischen Erkenntnisse nur ein wenig umschreiben und komprimieren muss, um eine adäquate Beratung in sprachlichen Zweifelsfällen zu liefern, verfehlt die Problemlage grundsätzlich. Denn vor der Abfassung der konsultativen Normtexte muss eine präzise Ermittlung der ursprünglichen Fragenkomplexe sowie eine ebenso genaue Identifikation derjenigen Wissensformen und Tatsachen stehen, die tatsächlich als angemessene, realistische Antworten auf die gestellten Fragen gelten können. Auch hier ist wieder die Verschränkung von systemlinguistischer Information und pragmatisch-

35 Entsprechende Untersuchungsperspektiven sind auch anschlussfähig an die sprachlichen Entwicklungstendenzen, die traditionell mit dem Begriff der (sprachlichen) Motivation ins Auge gefasst werden, vgl. Klein 2007. 
sozialsymbolischer Relevanz der Bewältigung von sprachlichen Zweifelsfällen zu beachten. Entsprechende Vorarbeiten für ein solches umfassendes Programm fehlen, soweit ich im Moment sehe, vollständig. Angesichts der technischen Möglichkeiten, die mittlerweile im Raum stehen, sollte auch eine frei zugängliche Internet-Plattform realisierbar sein, die erschöpfend über die Existenz und die Klärung der gegenwärtigen Zweifelsfälle im Deutschen Auskunft gibt. ${ }^{36}$

Nimmt man das gesamte Feld der Zweifelsfälle und die üblichen Annäherungen der Sprachenwissenschaft an dieses Thema in den Blick, so kann man - vielleicht etwas überspitzt und möglicherweise auch leicht blauäugig behaupten, dass hier etwas ganz besonderes auf der Kippe steht: nämlich der Status der Sprachwissenschaft als einer Disziplin, die nicht nur eskapistisch und theoretisch-abstrakt um sich selbst kreist, sondern die sich nachhaltig gegenüber den gesellschaftlich relevanten Sprachthematisierungen öffnet und aktiv das öffentliche Sprachbewusstsein und die gesellschaftlichen Sprachdiskurse mitgestaltet.

\section{Literatur}

Aarts, Bas (2007): Syntactic Gradience: the Nature of Grammatical Indeterminacy. Oxford. Ágel, Vilmos (2008): Bastian Sick und die Grammatik. Ein ungleiches Duell. In: Info DaF $35 / 1$, S. $64-84$.

Ammon, Ulrich u.a. (2004): Variantenwörterbuch des Deutschen. Berlin/New York.

Andersson, Lars G./Peter Trudgill (1990): Bad Language. Cambridge.

Antos, Gerd (1996): Laien-Linguistik. Studien zu Sprach- und Kommunikationsproblemen im Alltag. Am Beispiel von Sprachratgebern und Kommunikationstrainings. Tübingen.

Bellmann, Günter (2002): Standardisierung und Umstandardisierung: Der siebentelder siebte. In: Zeitschrift für Dialektologie und Linguistik LXIX Jg., H. 1, S. 1-37.

Biere, Bernd Ulrich/Hoberg, Rudolf (Hg.) (1995): Bewertungskriterien in der Sprachberatung. Tübingen.

Bredel, Ursula (2006): Orthographische Zweifelsfälle (Basisartikel). In: Praxis Deutsch 33. Jg. (Juli 06) [Thema: Orthographische Zweifelsfälle], S. 6-15.

Bünting, Karl-Dieter/Pospiech, Ulrike (1996): Das Sprachtelefon: Beobachtungen von Unsicherheiten und Unzufriedenheiten von Sprachteilhabern. Ein Werkstattbericht. In: Böke, K. u. a. (Hg.): Öffentlicher Sprachgebrauch. Opladen, S. 121-127.

Cherubim, Dieter (1983): Sprachentwicklung und Sprachkritik im 19. Jahrhundert. Beiträge zur Konstitution einer pragmatischen Sprachgeschichte. In: Cramer, Th. (Hg.): Literatur und Sprache im historischen Prozess. Sprache. Bd. 2. Tübingen, S. 170-188.

Cherubim, Dieter (2001): Pathologia linguae. Die Krankheiten der Sprache und deren Remedur. In: Burkhardt, A./Cherubim, D. (Hg.): Sprache im Leben der Zeit. Helmut Henne zum 65. Geburtstag. Tübingen, S. 427-447.

$36 \mathrm{Vgl}$. dazu insbesondere die bereits existierende IDS-Plattform „Grammatik in Fragen und Antworten" unter dem grammatischen Informationssystem grammis (http:// hypermedia.ids-mannheim.de/index.html). 
Cherubim, Dieter/Walsdorf, Ariane (2004): Sprachkritik als Aufklärung. In: Mittler, E. (Hg.): Die Deutsche Gesellschaft in Göttingen im 18. Jahrhundert. Göttingen.

Cölfen, Hermann (1996): Das Sprachtelefon an der Universität-GH-Essen. Ein Modell für ein neues Arbeitsfeld im Bereich der Linguistik? In: OBST 53, S. 9-21.

Davies, Winifred V. (2001): Standardisation and the School: Norm Tolerance in the Educational Domain. In: Linguistische Berichte 188, S. 393-414.

Davies, Winifred V./Langer, Nils (2006): The Making of Bad Language. Lay Linguistic Stigmatisations in German: Past und Present. Frankfurt/M.

Dieckmann, Walther (Hg.) (1989): Reichthum und Armut deutscher Sprache. Reflexionen über den Zustand der deutschen Sprache im 19. Jahrhundert. Berlin/New York.

Dieckmann, Walther (1991): Sprachwissenschaft und öfentliche Sprachdiskussion - Wurzeln ihres problematischen Verhältnisses. In: Wimmer, R. (Hg.): Das 19. Jahrhundert. Berlin/New York, S. 355-373.

Dieckmann, Walther (2006): Zum sprachlichen Umgang mit möglicherweise täuschenden Eindrücken. In: Muttersprache H. 1, S. 1-17.

Donalies, Elke (2003): Gebt endlich die Wortbildung freil Über unsinnige und sinnige Kritik an der Wortbildung. In: Sprachreport 1/2003, S. 26-32.

Dückert, J./Kempcke, G. (1989): Wörterbuch der Sprachschwierigkeiten. Zweifelsfälle, Normen und Varianten im gegenwärtigen deutschen Sprachgebrauch. (3. Auf1.) Leipzig.

Duden (2007): Richtiges und gutes Deutsch. Wörterbuch der sprachlichen Zweifelsfälle. (6. Aufl.) Mannheim u.a.

Eisenberg, Peter (2002a): Morphologie und Distribution - Zur Morphosyntax von Adjektiv und Adverb im Deutschen. In: Schmöe, F. (Hg.): Das Adverb. Zentrum und Peripherie einer Wortklasse. Wien, S. 62-76.

Eisenberg, Peter (2002b): Es gibt Gerïchte über eine Rettung Österreichs. Zur Rekonstruktion der Grammatik von Karl Kraus. In: Haß-Zumkehr, U. u.a. (Hg.): Ansichten der deutschen Sprache. Festschrift für Gerhard Stickel zum 65. Geburtstag. Tübingen, S. $55-87$.

Eisenberg, Peter (2007): Sprachliches Wissen im Wörterbuch der Zweifelsfälle. Über die Rekonstruktion einer Gebrauchsnorm. In: Aptum. Zeitschrift für Sprachkritik und Sprachkultur, S. 209-228.

Engel, Eduard (1918): Gutes Deutsch. Ein Führer durch Falsch und Richtig. Leipzig.

Fanselow, Gisbert/Féry, Caroline (2002): Ineffability in Grammar. In: Linguistische Berichte, Sonderheft 11. Hamburg, S. 265-307.

Fanselow, Gisbert u.a. (2006): Gradience in Grammar. Generative Perspectives. Oxford.

Frilling, Sabine (2004): Die Sprachberatung der GfdS. Resultate einer aktuellen Untersuchung. In: Der Sprachdienst H. 2, S. 42-49.

Frilling, Sabine (2005): Die Sprachberatung der GfdS (II). Auswertung der Anfragen von November 2004 bis Januar 2005. In: Der Sprachdienst H. 2-3, S. 69-77.

Glaser, Elvira (1992): Umbau partitiver Strukturen in der Geschichte des Deutschen. In: Sprachwissenschaft 17, S. 113-132.

Götz, Ursula (1995): Regionale grammatische Varianten des Standarddeutschen. In: Sprachwissenschaft 20, S. 222-238.

Grimm, Jacob (1818): Deutsche Grammatik, Vorrede. In: ders.: Jacob und Wilhelm Grimm über das Deutsche. Reiher, R. (Hg.), Leipzig: 1986, S. 137-153.

Heringer, Hans Jürgen (1984): Gebt endlich die Wortbildung freil In: Sprache u. Literatur in Wiss. u. Unterricht 53, S. 43-53.

Höhne, Steffen (1990): Kommunikationsberatung Deutsch als Fremdsprache. In: Sprache und Literatur in Wissenschaft und Unterricht 65, S. 84-95. 
Höhne, Steffen (1991): Sprachnorm und Sprachnormwandel als konstitutive Faktoren wissenschaftlicher Sprachberatung. In: Muttersprache 101, S. 193-217.

Joeres, Rolf (1996): Der Friede oder der Frieden. Ein Normproblem der Substantivflexion. In: Sprachwissenschaft 21/3, S. 301-336.

Klein, Wolf Peter (2003/2004) (Hg.): Sprachliche Zweifelsfälle. Theorie und Empirie. http://www.linguistik-online.de/16_03/index.html

Klein, Wolf Peter (2003/2004): Sprachliche Zweifelsfälle als linguistischer Gegenstand. Zur Einführung in ein vergessenes Thema der Sprachwissenschaft. In: Linguistik online 16. http://www.linguistik-online.org/16_03/klein.html

Klein, Wolf Peter (2003): In dubio contra reum? Zur Geschichte der Reflexion über sprachliche Zweifelsfälle. In: tribüne. Zeitschrift für Sprache und Schreibung, H. 2, S. 4-14.

Klein, Wolf Peter (2004a): Deskriptive statt präskriptiver Sprachwissenschaft!? Über ein sprachtheoretisches Bekenntnis und seine analytische Präzisierung. In: Zeitschrift für germanistische Linguistik 32, S. 376-405.

Klein, Wolf Peter (2004b): Koordination als Komplikation. Über eine strukturelle Ursache für die Existenz syntaktischer Zweifelsfälle. In: Deutsche Sprache 32, S. 357-375.

Klein, Wolf Peter (2006): Vergebens oder vergeblich? Ein Analysemodell zur Untersuchung sprachlicher Zweifelsfälle. In: Breindl, E./Gunkel, L./Strecker, B. (Hg.): Grammatische Untersuchungen. Analysen und Reflexionen. Tübingen, S. 581-599.

Klein, Wolf Peter (2007): Reflektierte Motivation. Überlegungen zu ihrer Rolle in Sprachgeschichte und Sprachwandel. In: Gallmann, P./Lehmann, Ch./Lühr, R. (Hg.): Sprachliche Motivation - zur Interdependenz von Inhalt und Ausdruck. Tübingen, S. 81-103.

Kolde, Gottfried (1976): Sprachberatung: Motive und Interessen der Fragensteller. In: Muttersprache 86, S. $20-47$.

Kolde, Gottfried (1980): Sprachpflege als angewandte Sprachwissenschaft. In: Der Sprachdienst XXIV, S. 97-106.

Konopka, Marek (1996): Strittige Erscheinungen der deutschen Syntax im 18. Jahrhundert. Tübingen.

Kühn, Ingrid (2003): Sprachberatung als Hilfeleistung im Identifikationsprozess. In: Janich, N./Thim-Mabrey, Ch. (Hg.): Sprachidentität - Identität durch Sprache. Tübingen, S. 91-105.

Kühn, Ingrid/Almstädt, Klaus (1997): Rufen Sie uns an - Sprachberatung zwischen Sprachwacht und Kummertelefon. In: Deutsche Sprache 25, S. 195-206.

Linke, A./Ortner, H./Portmann-Tselikas, P.R. (Hg.) (2003): Sprache und mehr. Ansichten einer Linguistik der sprachlichen Praxis. Tübingen.

Linke, Angelika (1991): Zum Sprachgebrauch des Bürgertums im 19. Jahrhundert. Überlegungen zur kultursemiotischen Funktion des Sprachverhaltens. In: Wimmer, R. (Hg.): Das 19. Jahrhundert. Berlin/New York, S. 250-281.

Linke, Angelika (1996): Sprache und Bürgertum. Zur Mentalitätsgeschichte des 19. Jahrhunderts. Stuttgart.

Lühr, Rosemarie (1992): Gleichartigkeit, Vollständigkeit, Vermeidung von Redundanz. Prinzipien von Sprachbewertungen im 19. Jahrhundert. In: Muttersprache 102, S. 341-358.

Mackowiak, Klaus/Steffen, Karin (1991): Statistische Auswertung der Anfragen an das Grammatische Telefon. In: Diskussion Deutsch 121, S. 518-535.

Maitz, Péter/Elspaß, Stephan (2007): Warum der „Zwiebelfisch“ nicht in den Deutschunterricht gehört. In: Info DaF 34/5, S. 515-530.

Mattheier, Klaus J. (1991): Standardsprache als Sozialsymbol. Über kommunikative Folgen gesellschaftlichen Wandels. In: Wimmer, R. (Hg.): Das 19. Jahrhundert. Sprachgeschichtliche Wurzeln des heutigen Deutsch. Berlin/New York, S. 41-72. 
Matthias, Theodor (1892): Sprachleben und Sprachschäden. Ein Führer durch die Schwankungen und Schwierigkeiten des deutschen Sprachgebrauchs. Leipzig.

Meinunger, André (2008): Sick of Sick? Ein Streifzug durch die Sprache als Antwort auf den „Zwiebelfisch“. Berlin.

Meyer, Kerstin 1993: Wustmanns Sprachdummheiten. Untersuchungen zu einem Sprachratgeber des 19. Jahrhunderts. In: Sprachwissenschaft 18, S. 223-315.

Moulin-Fankhänel, Claudine (2000): Varianz innerhalb der Nominalgruppenflexion. Ausnahmen zur sogenannten Parallelflexion der Adjektive im Neuhochdeutschen. In: Germanistische Mitteilungen. Zeitschrift für deutsche Sprache, Literatur und Kultur 52, S. 73-97.

Müller, Gerhard (1998): Normen und Normbedürfnisse aus Sicht der Sprachberatung. In: Neuland, E. (Hg.): Sprachnormen. (= Der Deutschunterricht 3/1998), S. 61-67.

Muthmann, Gustav (1994): Doppelformen in der deutschen Sprache der Gegenwart. Studie zu den Varianten in Aussprache, Schreibung, Wortbildung und Flexion. Tübingen.

Peyer, Ann/Portmann, Paul R. (1996): Norm, Moral und Didaktik - Die Linguistik und ihre Schmuddelkinder. Eine Aufforderung zur Diskussion. Tübingen.

Reis, Marga (1979): Ansätze zu einer realistischen Grammatik. In: Grubmüller, K. u.a. (Hg.).: Befund und Deutung. Tübingen, S. 1-21.

Sanders, Daniel (1872): Wörterbuch der Hauptschwierigkeiten in der deutschen Sprache. Berlin.

Schneider, Jan Georg (2005): Was ist ein sprachlicher Fehler? Anmerkungen zu populärer Sprachkritik am Beispiel der Kolumnensammlung von Bastian Sick. In: Aptum. Zeitschrift für Sprachkritik und Sprachkultur 2, S. 154-177.

Scholze-Stubenrecht, Werner (1991): Die Sprachberatungsstelle der Dudenredaktion. In: Deutsche Sprache 19, S. 178-182.

Schrodt, Richard (1995): Warum geht die deutsche Sprache immer wieder unter. Die Problematik der Werthaltungen in der deutschen Sprache. Wien.

Schütze, Carson T. (1996): The Empirical Base of Linguistics. Grammaticality Judgements and Linguistic Methodology. Chicago/London.

Siebenborn, Elmar (1976): Die Lehre von der Sprachrichtigkeit und ihren Kriterien. Studien zur antiken normativen Grammatik. Amsterdam.

Stenschke, Oliver (2007): Ende diesen Jahres. Die Flexionsvarianten von Demonstrativpronomina als ein Beispiel für Degrammatikalisierung. In: Deutsche Sprache 35, S. $63-85$.

Thieroff, Rolf (2003): Die Bedienung des Automatens durch den Mensch. Deklination der schwachen Maskulina als Zweifelsfall. In: Linguistik online 16, 4/2003 http://www. linguistik-online.de/16_03/thieroff.html

Wahrig (2003): Fehlerfreies und gutes Deutsch. Das zuverlässige Nachschlagewerk zur Klärung sprachlicher Zweifelsfälle. Gütersloh/München.

Wolf-Bleifuß, Brigitte (2004): Sprachberatung und Wörterbuchbenutzung. In: Scharnhorst, J. (Hg.): Sprachkultur und Lexikographie. Von der Forschung zur Nutzung von Wörterbüchern. Frankfurt/M., S. 247-280.

Wustmann, Gustav (1891): Allerhand Sprachdummheiten. Kleine deutsche Grammatik des Zweifelhaften, des Falschen und des Häßlichen. Ein Hilfsbuch für alle, die sich öffentlich der deutschen Sprache bedienen. Leipzig. 